\title{
Concept and Construction of an Interactive Folktale System
}

\author{
Kozi Miyazaki, Yurika Nagai, Takenori Wama, and Ryohei Nakatsu \\ Kwansei Gakuin University, School of Science and Technology \\ 2-1 Gakuen, Sanda 669-1337, Japan \\ miyazaki@nirvana.ne.jp, \\ \{bfy68428, scbc0057, nakatsu\}@ksc.kwansei.ac.jp
}

\begin{abstract}
In this paper as one example of a new edutainment system, we propose an "Interactive Folktale System," which makes it possible for people, especially children, to enjoy folktales in various ways. They can appreciate the generation of original tales and variations. Also they can interact with the story by communicating with its characters or by playing one of the characters. Moreover, they can easily create a new folktale by changing the original story or by describing a new story.
\end{abstract}

\section{Introduction}

Recently, extremely rapid progress in computer graphics (CG) technology has made it possible to virtually generate various kinds of objects in the real world. Such technologies are being widely applied to video games such as Role Playing Games (RPG). In RPGs, by controlling one of the game's computer graphic characters (CG characters), people can enjoy the development of fantasies, myths, and folktales. The problem with present RPGs is that their main focus is "fighting enemies," thus neglecting the original capability of fantasies or myths. On the other hand, the original function of fantasies, myths, and folktales was to influence the imagination of readers and cultivate creative capabilities.

There are several reasons why present RPGs avoid this important issue. One is that it is easier to sell and market games that directly appeal to such basic desires as fighting and conquering enemies rather than to highly sophisticated, imaginative, and/or creative capabilities. Another is that it is becoming increasingly difficult and time-consuming to develop/create contents and programs related to CG, and in this area non-professionals face barriers to develop entire systems from scratch.

Based on the above considerations, focusing on Japanese folktales we are developing an "Interactive Folktale System" with which people can enjoy various story developments. For example, they can enjoy original Japanese folktales as animation. Also using the system's interactive capabilities, they can interact with the characters in a story such as chatting with them. Also by controlling one of the characters as their avatar, they can enjoy the development of their own story. In addition users can reveal their own creative power by generating variations based on the original story, by creating a new story by combining multiple stories, and even by 
creating a completely new story that describes a new script using a script language supplied by the system. The last function of this system is crucial because the creation of new stories strengthens the people's creative capabilities.

We will first describe the basic concept of this system. Also we will explain the kinds of databases we are preparing. Since a key issue is an authoring system that makes it possible for an interactive folktale system to be realized, we will describe the details of the authoring system.

\section{Related Works}

One of the most important issues for interactive stories is controlling the development of interactive stories that have been actively studied as "Interactive Storytelling" $[1][2][3][4]$. The key issues in interactive storytelling include the generation of autonomous interactive characters and the emergence of storylines based on interactions between virtual characters and users and also among virtual characters. This is undoubtedly the final target of interactive storytelling. The problem with present interactive storytelling is that, since the generation of sophisticated autonomous characters is so complicated, it is difficult to maintain the consistency of the generated story for more than several minutes [4]. Game and e-learning users are expected to interact with the system for hours. Therefore, for these applications, developing a narrative based on a plot prepared beforehand is more realistic. If many story variations must be generated, our idea is to produce a story by connecting various short stories called 'segments.' In this case there must be an authoring system with which content creators can easily develop interactive contents.

\section{Interactive Folktale System}

\subsection{Basic Concept}

We are developing a system where having a database of Japanese folktales, it is possible to interactively regenerate original stories, generate wide variations based on the original stories, and even generate new stories [5]. For this purpose it is necessary to develop a model of folktale generation. To analyze and model folktales, V. Prop's study [6] is one of the best known. In this book, based on the analysis of old Russian magic folktales, he insisted that each folktale be considered a series of short story segments (hereafter, segments). Each segment could be classified into one of 31 categories called a "function" that corresponds to the kind of action of characters in the segment. Also he found that almost all folktales are constructed based on only one story line, in other words, one specific sequence of segments.

Studying whether this basic concept can be applied to other folktales and even to general stories is an interesting research theme. We applied his concept to Japanese folktales and also tried to develop an interactive folktale generation system.

Based on the concept of Prop, our hypothesis is the following:

(1) Each Japanese folktale can be segmented into a series of short story segments, as shown in Fig. 1. 


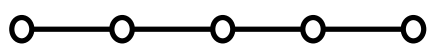

Fig. 1. Story as a sequence of story segments

(2) The sequence of these segments defines a specific story.

(3) Each segment is classified into one of several functions, each of which is defined based on the actions of the characters in it.

\subsection{Analysis of Japanese Folktales}

Based on the above hypothesis we analyzed fifty Japanese folktales and found that about fifty functions are enough to cover the stories in Japanese folktales. The number of functions is larger than in Prop's study because Japanese folktales cover wider areas than Russian magic folktales, and also some of them could be merged based on further research. We can treat Japanese folktales following the method proposed and adopted by Prop.

\subsection{Functions of the System}

Based on the above considerations, we are going to develop a system that can treat Japanese folktales in various ways. The major users of this system are children. As for video games we introduce the capability of interactive functions between the system and users to the system. We expect that users will enjoy the following methods of system interaction.

(1) Watching the generation of animation for each of the original Japanese folktales.

(2) Changing the characters as well as the backgrounds to enjoy wide variations of the original stories.

(3) Generating new stories. For example, it will be possible to create a new story by combining multiple original stories and to create a completely new story using a script language dedicated for the system.

(4) Watching the animations and talking to the characters in the stories or even controlling one as an avatar.

The interactive function described in (4) has been already realized in video games. For video games, however, all possible variations must be defined and involved in the system beforehand. So the development process of video games is time-consuming and expensive. We have developed an authoring system that supports the content development process of these interactive contents.

\subsection{System Construction}

The system consists of a folktale database and authoring system.

\subsubsection{Folktale Database}

The database contains various kinds of data necessary to handle Japanese folktale generation based on three-dimensional computer graphics (3D-CG). It consists of the following sub-databases: 
(1) Story segment database

This database stores a set of short segments extracted from Japanese folktales. Each segment defines an event in it and is expressed as a script using the script language dedicated to this system. Since the language makes it possible to develop an interactive story, users can interactively enjoy story development.

(2) Character/background database

Various characters, objects, and backgrounds that appear in Japanese folktales are stored here. Using this database, users can change characters or backgrounds and enjoy new sensations even if watching the same story. Also users can create a new story by combining the data and the script language.

(3) Animation database

In folktales, characters achieve different kinds of behaviors. It is very troublesome to prepare new animations that correspond to these behaviors when required by the story. Therefore by analyzing Japanese folktales we examine the kind of animations that can be generally used to create folktales and store these animations in the database. Thus users can create any kind of CG animation by simply choosing a character and animation from this database.

\subsubsection{Authoring System}

The authoring system has the following two functions:

(1) Event generation

Each segment corresponds to a scene in a movie or a play. Therefore the authoring system must have a function that generates any kind of event and character behaviors in the scene. For this purpose we developed a script language. One example of such a script language is TVML [7]. For TVML, users select characters and backgrounds stored in the database, and then they can create a CG scene by defining the actions of the characters using TVML. However, this system lacks an interactivity function. But since our developed script language has an interactivity function, users can describe interactive events in each segment.

(2) Story generation

The system must generate a story by selecting segments one by one and generating an event in each segment, Here the essential issue is the algorithm for selecting a sequence of segments to generate adequate story. The details will be described in the next section.

\section{Story Generation Based on Segment Concatenation}

\subsection{Basic Concept}

The sequence of selected segments obviously defines the story. Therefore developing an algorithm that defines the selection process is necessary. This is an essential problem in story generation and remains a difficult problem. In our study, so far the following basic methods have been adopted:

(1) Generation of an original story

As basic information, the sequence of segments for each original folktale is stored in the database. Using this information, users can enjoy the story development of each 
original Japanese folktale. Even in this case, users can enjoy variations by changing the characters and/or the backgrounds of the original story.

(2) Story transition from a story to a different story

Japanese folktales contain wider story variations than Russian magic folktales. However there are many cases where the short story within a segment resembles a short story from a different segment. For example, a scene where a boy called Momorato leaves his home to fight monsters resembles a scene where a boy called Issun-Bohshi leaves his home for the big city. In other words, these two segments have identical functions. Therefore we introduce a function that jumps from one story to another when these two stories have segments with identical functions. Figure 2 illustrates the story jump. This mechanism makes it possible to generate a huge amount of variety in story development.

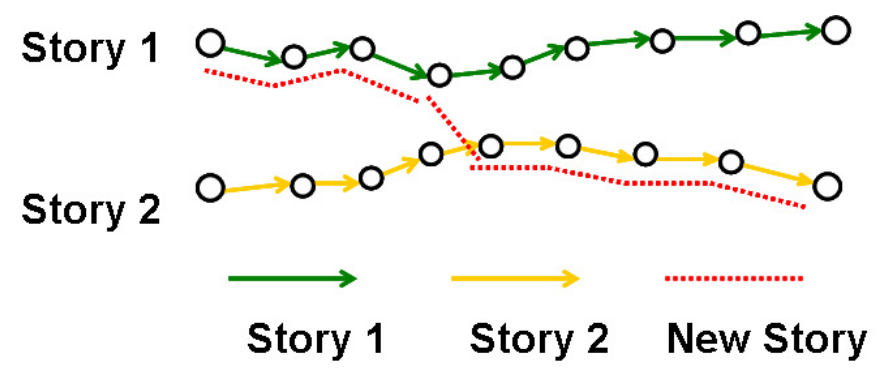

Fig. 2. Story transition

\subsection{Segments and Stories}

Let $\left\{S_{i}\right\}(i=1,2, \ldots)$ be a set of stories. Also let $\left\{\mathrm{SE}_{\mathrm{i}}\right\}(\mathrm{i}=1,2, \ldots)$ be a set of story segments. Then each story can be expressed by a series of story segments:

$\mathrm{Si}=\mathrm{SE}_{\mathrm{i} 1}, \mathrm{SE}_{\mathrm{i} 2}, \mathrm{SE}_{\mathrm{i} 3}, \ldots$

\subsection{Segment Index}

We define each segment $\mathrm{SE}_{\mathrm{i}}$ by the following index:

Function: $F_{i}$

Main character: $\left(\mathrm{MC}_{\mathrm{i} 1}, \mathrm{MC}_{\mathrm{i} 2}, \ldots\right)$

Sub character; $\left(\mathrm{SC}_{\mathrm{i} 1}, \mathrm{SC}_{\mathrm{i} 2}, \ldots\right)$

The characters are listed based on order of importance.

\subsection{Concatenation}

(1) Let $\mathrm{D}_{\mathrm{ij}}$ be a distance between $\mathrm{SE}_{\mathrm{i}}$ (function: $\mathrm{F}_{\mathrm{i}}$ ) and $\mathrm{SE}_{\mathrm{j}}$ (function: $\mathrm{F}_{\mathrm{j}}$ )

Example: $\mathrm{D}_{\mathrm{ij}}=0\left(\right.$ where $\mathrm{F}_{\mathrm{i}}=\mathrm{F}_{\mathrm{j}}$ )

$$
D_{i j}=1\left(\text { where } F_{i} \text { does not equal } F_{j}\right)
$$

(2) Let two stories $S_{i}$ and $S_{j}$ be

$\mathrm{S}_{\mathrm{i}}=\mathrm{SE}_{\mathrm{i} 1}, \mathrm{SE}_{\mathrm{i} 2}, \ldots ., \mathrm{SE}_{\mathrm{im}}, \ldots \ldots$.

$\mathrm{S}_{\mathrm{j}}=\mathrm{SE}_{\mathrm{j} 1}, \mathrm{SE}_{\mathrm{j} 2}, \ldots \ldots \mathrm{SE}_{\mathrm{jn}}, \ldots \ldots$ 
If the distance between $\mathrm{SE}_{\mathrm{im}}$ and $\mathrm{SE}_{\mathrm{jn}}$ is less than a predetermined threshold $\mathrm{T}$, then story $S_{i}$ could jump to $S_{j}$ at $S_{\text {im }}$ like:

$\mathrm{SE}_{\mathrm{im}-1}, \mathrm{SE}_{\mathrm{jn}}, \mathrm{SE}_{\mathrm{jn}+1}, \ldots$,

Example: If the distance between two segments is defined by (4.3), then the threshold is set to $\mathrm{T}=0$. This means that if there is a segment whose function is identical to another segment, then at this point, the story can jump to another story.

\subsection{Maintaining Consistency in a Story}

Consistent story development must be maintained even though a story jump might occur. Consistency contains various issues. For simplicity, however, here only the consistency regarding the $\mathrm{CG}$ models of characters is considered. If segment $\mathrm{SE}_{\mathrm{im}}$ is replaced by another segment $\mathrm{SE}_{\mathrm{in}}$, the consistency of the characters can be retained as follows. All the characters in $\mathrm{SE}_{\mathrm{jn}}$ corresponding to the number of characters in $\mathrm{SE}_{\mathrm{im}}$ are replaced. If several characters remain in $\mathrm{SE}_{\mathrm{in}}$, they remain unchanged. This operation is carried out both for main and sub characters.

\section{Authoring System}

We developed an authoring system that supports the realization of the interactive folktale system described above. In it, there are two kinds of files: object (Object.txt) and script (Script.txt). The system generates interactive narrative by interpreting these files.

Script.txt: A text file that defines the generation of each scene, the actions of each character, and the interactions between users and the characters and also among characters.

Objext.txt: A text file used for the basic definition and setting of 3D objects.

Figure 3 shows an example of the basic structure of Script.txt. An example of Object.txt is shown in Fig. 4. Since the details of this authoring system are described in [5], a detailed description is omitted here.

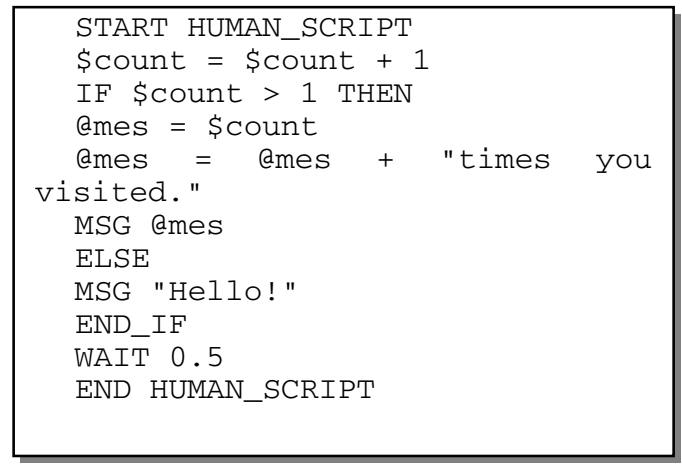

Fig. 3. Basic structure of Script.txt 


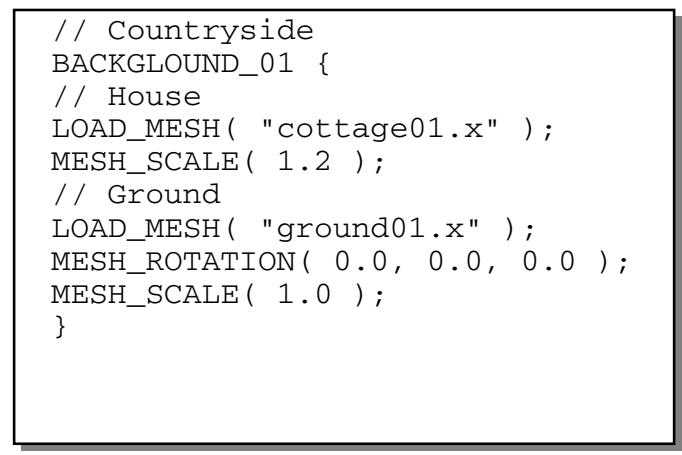

Fig. 4. A description sample of Objext.txt

\section{System Evaluation}

We evaluated the functions of the Interactive Folktale System by selecting five representative folktales for experiments on which we carried out the following processes.

(1) Segmented each story into a set of short segments and generated a segment database.

(2) Generated CG models necessary to generate scenes and objects for all segments.

(3) Generated animation data necessary to generate actions and events that occur in each short story.

After the above preparation processes, we carried out story generation, which was confirmed in experiments.

(1) Generation of animation: All five classic tales were generated as an animated movie by concatenating segments based on the original story. Adding a slight variation to the animation by changing character models or backgrounds gave users a fresh feeling each time they observed the animation.

(2) Automatic story transition: Then we tested the story transition function described in Section 4. At certain segments when other segments with the same function are found, the story randomly transits to another segment, changing the story line. Although further study is still necessary, such story transition capability is very powerful and is a key function for the automatic generation of stories. Figure 5 illustrates an example of such a story transition. In one story, Princess 'Kaguya' who was born in a bamboo grows up to be a beautiful lady and attracts many noble men. In another story 'Momotaro' who was born in a peach grows up and leaves home to fight monsters. Since the maturation scenes match, the story starts as Kaguya and then changes to Momotaro. Figure 5 shows Princess Kaguya leaving her home to go to monster island. 


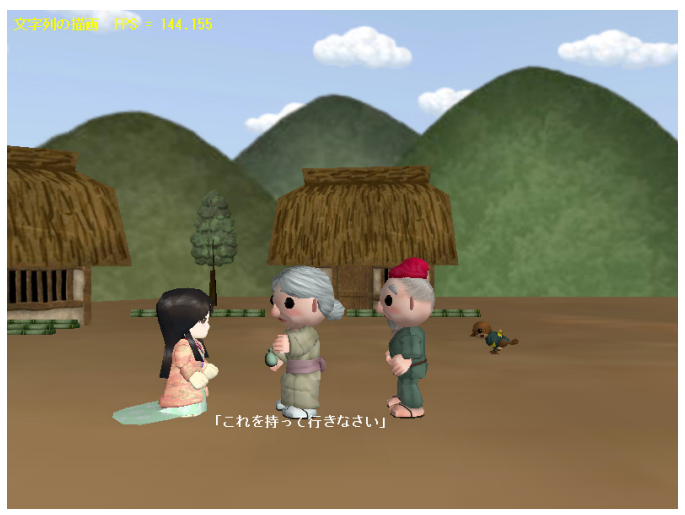

Fig. 5. Example of a story transition

Figure 6 shows several other scenes in this story.
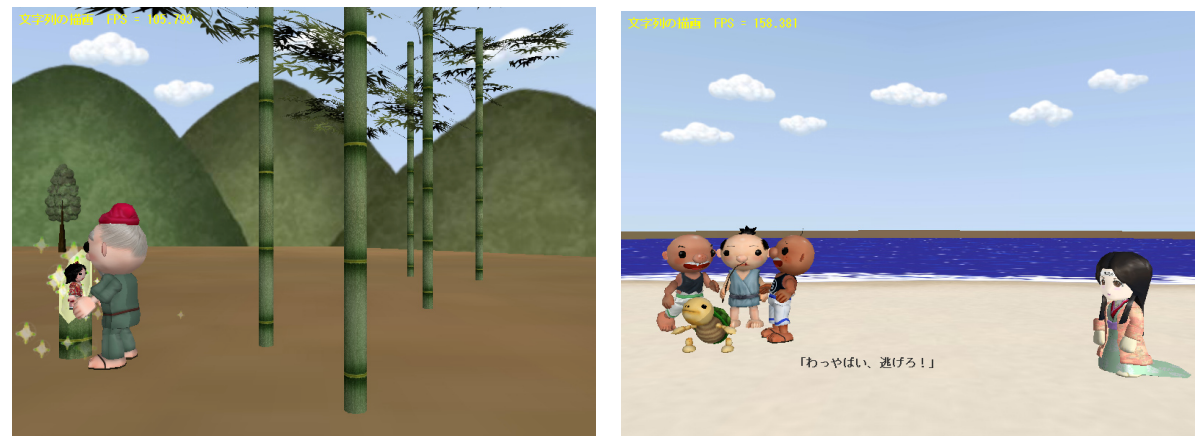

Fig. 6. Examples of scenes in the system

\section{Conclusion}

In this paper we proposed an Interactive Folktale System that can generate various types of Japanese folktales based on the concept of Prop. The system consists of an authoring system and three types of databases: story segment, CG, and animation. Taking Japanese folktales as an example, we constructed the system and carried out evaluation experiments that showed the system can generate original folktales, their variations, and also new stories by jumping from one story to another.

There are several issues to be pursued further. The most important one is to strengthen its interactive capability. Although the system basically has the function of interactivity, so far we have focused on the regeneration of original stories, generation of their variations, and the transition from one story to another. By extending these basic functions, we will develop the second prototype where story would change depending on the interaction between users and the system. 


\section{References}

1. Swartout, W., et al.: Toward the Holodeck: Integrating Graphics, Sound, Character and Story. In: Proceedings of the Autonomous Agents 2001 Conference (2001)

2. Mateas, M., Stern, A.: Socially Intelligent Agents: The Human in the Loop. In: AAAI Fall Symposium (2000)

3. Young, R.M.: Creating Interactive Narrative Structures: The Potential for AI Approaches. In: AAAI Spring Symposium in Artificial Intelligence and Interactive Entertainment. AAAI Press, Stanford, California, USA (2000)

4. Charles, F., Cavazza, M.: Exploring Scalability of Character-based Storytelling. In: Proceedings of ACM AAMAS'04 (2004)

5. Miyazaki, K., Nagai, Y., Bosser, A.G., Nakatsu, R.: Architecture of an Authoring System to Support the Creation of Interactive Contents. In: Harper, R., Rauterberg, M., Combetto, M. (eds.) ICEC 2006. LNCS, vol. 4161, pp. 165-174. Springer, Heidelberg (2006)

6. Prop, V.: Morphology of the Folktale. University of Texas Press (1968)

7. Douke, M., Hayashi, Makino, E.: A Study of Automatic Program Production Using TVML. In: Short Papers and Demos, Eurographics'99, pp. 42-45 (1999) 